\title{
PRAXIS DE UNA RED LOCAL DE GESTIÓN UNIVERSITARIA DEL CONOCIMIENTO Y LA INNOVACIÓN Y DESARROLLO LOCAL
}

\section{ROLE OF KNOWLWDGE AND INNOVATION UNIVERSITY MANAGEMENT LOCAL NETWORK IN LOCAL DEVELOPMENT}

\author{
Alexander Báez Hernández ${ }^{1}$, Carlos Alberto Hernández Medina²,», \\ Magdalys Alibet Carrasco Fuentes ${ }^{3}$
}

\begin{abstract}
RESUMEN
El objetivo es presentar el concepto de Red Local de Gestión Universitaria del Conocimiento y la Innovación (GUCID) como base de un Sistema de Innovación Local. La investigación se justifica porque la red es una forma de lograr, en lo local, economías exitosas y rendimientos crecientes. El reconocimiento creciente de las redes está sustentado en que, independientemente de la cantidad de conocimiento codificado que se produzca, si no hay relación entre la cantidad de conocimiento codificado y las competencias (conocimiento tácito) encarnadas en individuos y organizaciones, la localidad no captará el impulso productivo del conocimiento, ni lo plasmará en innovación siguiendo un sendero de desarrollo estable. La función del Centro Universitario Municipal en la Red es coordinar la innovación tecnológica como centro de pivote que conecta el conocimiento producido en la Red Universitaria con las necesidades locales para resolver sus problemas productivos y de servicios. Como metodología se utilizan los principios de la Educación Popular para introducir la ciencia que soluciona los problemas en Empresas Agropecuarias. La innovación, promovida por los estudiantes en su papel de "maestros ambulantes", logra la introducción de Resultados Científicos que aseguran la solución de los problemas productivos de las Unidades Agropecuarias.
\end{abstract}

Palabras Clave: Gestión Universitaria del Conocimiento, extensionismo, desarrollo local, conocimiento tácito.

\footnotetext{
${ }^{1}$ Master en Economía. Facultad de Ciencias Administrativas, Universidad Central de Ecuador, Quito, Ecuador. albaez@uce.edu.ec.

2Master en Agricultura Sostenible. Centro Universitario Municipal Camajuaní. Villa Clara. Cuba. cahm862@uclv.edu.cu. orcid.org/0000-0003-3446-3014

$3^{3}$ Master en Ciencias de la Educación. Centro Universitario Municipal Camajuaní. Villa Clara. Cuba. alibet7305@uclv.edu.cu,
} 


\section{ABSTRACT}

The objective is to present the concept of university knowledge management local networks like the base of a Local Innovation System. The investigation is justified because the network is a form of achieving, in a local environment, successful economies and growing yields. The growing recognition of networks it is sustained in idea that, independently of quantity of coded knowledge that takes place, if there is no relationship between quantity of coding knowledge and competitions (tacit knowledge) embodied in individuals and organizations, locality won't capture productive impulse of knowledge, neither it will capture it in innovation following a path of stable development. Municipal University Centre function, in a local network, is to coordinate technological innovation as a pivot that connects knowledge taken place in University network with local necessities, to solve production and services troubles. As methodology was used the principles of Popular Education to introduce science that solves problems in Agricultural Companies. Innovation, promoted by students in their paper of "traveling teachers", achieves the introduction of scientific results that assure the solution of productive problems of Agricultural Units.

Keywords: University Knowledge Management, extensionism, local development, tacit knowledge.

\section{INTRODUCCIÓN}

Las estrategias de desarrollo y las políticas de equilibrio territorial en América Latina, han estado sustentadas durante décadas en el paradigma del desarrollo polarizado, propio del industrialismo, donde la organización productiva responde a un esquema centralista, regido por un crecimiento planificado desde el gobierno nacional. Para ese enfoque tradicional, el desarrollo era posible gracias a la construcción de infraestructuras y la continua expansión de las empresas industriales localizadas preferentemente en los grandes centros urbanos. La tipología de la innovación tecnológica introducida y las políticas económicas, demandaban la consecución de economías de escala y externas. Así se diseñaron las políticas en Cuba, siguiendo la tendencia que entendía el desarrollo promovido desde arriba.

Desde la década del 90 se dio una situación de crisis y reemplazo de viejos paradigmas, pasando de la visión redistributiva al potencial de desarrollo en el territorio. Eso provocó un cambio significativo en la conceptualización de la política de desarrollo regional. La visión del desarrollo endógeno introduce como estrategia dominante el desarrollo difuso, desde abajo, en el que el espacio asume la connotación de factor estratégico para el desarrollo del territorio. El foco de análisis se trasladó de la problemática de la redistribución a la creación de recursos locales y la endogenización de los mecanismos de desarrollo.

Esto hace necesaria la construcción de entornos innovadores en cada territorio y marca la insuficiencia del enfoque tradicional del desarrollo, basada en factores productivos. Según Alburquerque (1998), las ventajas competitivas dinámicas no se basan tanto en la dotación de recursos naturales o salarios bajos, sino en la introducción de la innovación tecnológica y organizativa, o, en suma, de elementos de conocimiento.

El presente estadio de desarrollo es caracterizado por el paso gradual de un sistema pro- 
ductivo basado en la estandarización, a un sistema focalizado en la variedad y calidad de los productos y procesos productivos. Nuevos factores parecen haber reemplazado la tierra, el trabajo y el capital físico. Recursos naturales y medioambientales, recursos humanos y tecnología están convirtiéndose en elementos clave debido a la Revolución Tecnológica.

Es muy clara la importancia que tienen las relaciones que se establecen entre los actores y agentes que componen un sistema económico social como fuente para su desarrollo como sistema. Los vínculos entre las universidades, empresas y sectores cobran especial relevancia en un sistema cada vez más integrado, donde la internacionalización de la producción y del comercio es cada vez más importante. Si queremos insertarnos en senderos de crecimiento sostenido es fundamental que reconozcamos en dichos vínculos un factor clave para lograrlo (Cimoli, 2005).

El surgimiento de un sistema de redes como expresión de disposiciones institucionales e industriales para la difusión de los vínculos sistémicos entre actores locales es condición necesaria para avanzar por el sendero del desarrollo local sostenible. Por eso las redes son una categoría que adquiere cada vez mayor importancia para saber si un país, región o localidad es capaz de generar un sendero alternativo hacia el desarrollo sustentable.

La toma de decisiones en función del desarrollo en el ámbito local, regional y nacional, no está determinada exclusivamente por la presión en la demanda de productos o servicios. Responde también a factores especiales que provienen de la organización de los procesos productivos y sus rutinas dominantes. En el mundo globalizado los vínculos e interacciones entre esos factores han adquirido nuevo carácter y mayor importancia. Para los países subdesarrollados, exportadores de materia prima, esos vínculos son una estructura capaz de generar un sendero alternativo hacia el desarrollo. Algunas de las características del desarrollo de un país en período de crecimiento inducido por las exportaciones se pueden describir en función de los vínculos de sus productos principales (Hirschman, 1977).

Un elemento básico al analizar los Sistemas de Innovación es que los nexos e interacciones sistemáticos no sólo se refieren a las empresas sino también, en general, a los encadenamientos entre universidades, empresas, organizaciones y marcos institucionales que la sociedad genera como forma propia de organización social. Así, el concepto de redes puede considerarse una variable de observación para comprender si los encadenamientos y las interacciones sistemáticos se difunden realmente a los sistemas innovadores.

Las redes son una forma de incorporar vínculos e interacciones que hacen posible, en el nivel local, la existencia de economías exitosas y rendimientos crecientes. El reconocimiento cada vez mayor que tienen las redes está sustentado en la idea de que independientemente de la cantidad de información y conocimiento codificado que produzca una sociedad, si no hay vinculación entre la cantidad total de conocimiento codificado y las diversas competencias (conocimiento tácito) encarnados en los individuos y en las organizaciones, el país no será capaz de captar el impulso productivo del conocimiento y plasmarlo en innovación y en un sendero de desarrollo estable (Soete, 1996; Dosi, 1999; Cimoli \& De la Mothe, 2001).

El concepto de sistema de innovación no es suficiente, en el nivel local, para comprender las causas de la creciente brecha espacial y temporal que existe entre la generación del conocimiento y su difusión y uso para la innovación tecnológica en nuestros países subdesarrollados. Creemos que esta brecha está dada fundamentalmente por la dificultad existente para generar políticas que acerquen los intereses de los que producen conocimiento y los que deben utilizarlo en función de optimizar los procesos de producción de bienes materiales y servicios. 
Muchos autores reconocen a las empresas como actores principales de las redes porque están integradas a través de un gran complejo de vínculos con otras empresas e instituciones. La forma en la que una empresa se integra a una red aumenta su capacidad para captar conocimientos externos e incrementar sus rendimientos. En la producción capitalista eso pone de relieve el papel central de la competencia entre las empresas integradas en redes, en el sentido de rivalidad o pugna en el mercado, como el factor de mayor peso ale explicar la configuración de un sistema de innovación.

Los cambios estructurales de los sistemas de innovación de los países de América Latina han seguido un sendero que fue afectado intensamente por la globalización generalizada en lo económico. Una característica fundamental de la globalización es la intensidad y ritmo del proceso de liberalización y los incentivos introducidos por cambios en los precios internacionales que afectan radicalmente la estructura del mercado y el papel desempeñado por las empresas locales.

Ejemplo de eso es el poder de mercado de las subsidiarias de empresas multinacionales que determinan el perfil de producción local y desarrollan aún más las redes de conocimiento en los países desarrollados a costa de los subdesarrollados. Las fusiones y adquisiciones de empresas locales desechan y subutilizan recursos humanos y bienes de capital locales en interés de los capitales foráneos. Gracias a esas denigrantes relaciones económicas entre el Norte desarrollado y rico y el Sur pobre y subdesarrollado, la brecha tecnológica y de conocimiento no se reduce y se agrava a diario. Los efectos de esas relaciones, impuestas por el Norte a través de organismos financieros como el BID y el FMI, sobre los procesos competitivos y sobre las estructuras de mercado afectan los sistemas de innovación local y las redes locales de América Latina.

Ese proceso contribuyó a desarrollar aún más las redes de conocimiento en los países de economía más desarrollada a costa de nuestras economías, aumentando la brecha tecnológica y de conocimiento Norte-Sur. La internacionalización de la producción que se produce actualmente en las economías de América Latina es un factor que incrementa la demanda de conocimiento y de actividades en red, dirigida hacia las economías más desarrolladas en perjuicio de las más pobres.

La innovación se concibe como proceso interactivo y no incumbe a una empresa aislada. Exige un proceso de búsqueda activa para dar con nuevas fuentes de conocimiento y tecnología y poder aplicarlas a productos y procesos de producción de bienes y servicios. Es un proceso social que se desenvuelve mejor cuando se logra interacción entre proveedores y usuarios de bienes, servicios, conocimiento y tecnología. Incluye organizaciones e infraestructura pública como universidades y centros de investigación (Freeman, 1994; Nelson, 1998).

A nivel local, una red es un concepto social que... "pretende englobar a [...] las organizaciones y personas comprometidas con el sistema por diversos intereses, a las corporaciones dedicadas a la fabricación, a los laboratorios de investigación y desarrollo, a los bancos de inversión, las instituciones educativas y a los organismos reguladores" (Misa, 1991). A su vez, estos vínculos al interior de las redes están sustentados por una inversión cada vez mayor y por el carácter local de la mayoría de las actividades de aprendizaje, determinando las oportunidades y limitaciones a las que se enfrenta cada proceso de innovación y difusión de conocimiento.

Las empresas buscan vínculos para fomentar el aprendizaje interactivo entre ellas y que las redes y socios externos aporten activos complementarios. Esto ayuda a repartir entre un número mayor de organizaciones los costos y riesgos derivados de la innovación para acceder 
a los resultados de nuevas investigaciones, a adquirir componentes tecnológicos clave para productos o procesos nuevos y a compartir activos en los procesos de fabricación, marketing y distribución.

Las redes son una categoría muy importante para comprender y explicar el funcionamiento de los sistemas de innovación. Coordinan y regulan el papel complementario que desempeñan la cooperación, coordinación y competencia al analizar los patrones de cambio y el mejoramiento del desempeño económico. Cuando están organizadas de manera conveniente son un poderoso motor que estimula el progreso y el desarrollo económico. Cuando su organización y conectividad son deficientes pueden inhibir en gran medida el proceso de innovación.

La eficacia del sistema económico en la asignación de recursos a ciertas funciones económicas y en el desarrollo y difusión de innovaciones técnicas y organizacionales, depende cada vez más de la posibilidad de acceso al conjunto de nexos dentro de la red y del acceso a los flujos de conocimientos que se necesitan en un contexto institucional específico (Dosi et al., 1990).

Pertenecer a una red "...no sólo reduce el costo de la información sino que también evita ser objeto de ulteriores barreras de exclusión, reduce el costo del ingreso a la vez que minimiza los costos de egreso y limita las inversiones internas hundidas e irreversibles. Las redes permiten acceder al mismísimo proceso de fijación de normas, establecer trayectorias tecnológicas acordadas y reducir la repetición de investigaciones. Sin embargo, la posibilidad de compartir incertidumbres, riesgos y costos por medio de las redes sería insuficiente por sí sola para explicar por qué las redes de innovadores son tan persistentes, a menos que se espere que los beneficios de largo plazo compensen con creces los costos inmediatos de la cooperación. La explicación está en la expectativa de obtener cuantiosas ganancias conjuntas a partir de la acumulación conjunta de know-how tecnológico y de una aceleración conjunta y significativa de los plazos de entrega de productos o servicios superiores. Ese aspecto ha sido estudiado mucho menos." (De Bresson \& Amesse, 1991).

La competencia y la eficiencia en nuestro mundo globalizado no están generadas por empresas que actúan en forma aislada, sino por redes constituidas por organizaciones disímiles. Las empresas deben adaptarse a los rápidos cambios de las exigencias del mercado y tomar la delantera innovando sus productos y procesos de producción en un mundo en el que el desarrollo tecnológico tiene un ritmo cada vez mayor. Este elemento hace que a las empresas les resulta cada vez más difícil generar todo el conocimiento pertinente en forma individual para traducirlo luego en productos o procesos de producción innovadores. Para reducir los riesgos y acortar el tiempo que lleva poner un producto en el mercado, tienen que especializarse y para tener éxito en el proceso de innovación se apoyan cada vez más en el conocimiento y know how complementario que aportan otras empresas.

El auge de las Tecnologías de la Información y las Comunicaciones constituye un ambiente nuevo. Está caracterizado por la incertidumbre tecnológica pero implica nuevas oportunidades y desafíos para operar en redes. La aparición de nuevas tecnologías tiene consecuencias importantes para la economía de los países de América Latina.

El conocimiento tácito y local desempeña un papel fundamental en la mayoría de las actividades de innovación. El proceso de innovación tiene dos aspectos en común con el conocimiento: uno es que es un elemento público en potencia, y el otro es que es un elemento tácito. El elemento público consiste en el conocimiento asequible que sólo en potencia está al alcance de quien lo procura, puesto que existen distintas formas de conceptualizarlo y de codificarlo. El elemento tácito surge de un concepto desarrollado por Polanyi (1967), diciendo 
que estaba relacionado con "....aspectos del conocimiento y de la percepción intuitiva y con otras aptitudes propias de los individuos, que están mal definidas, no codificadas o no tienen estado público y que, por lo tanto, ni siquiera los propios individuos pueden expresar en su totalidad; aptitudes estas que difieren de una persona a otra, pero que pueden ser compartidas en gran medida entre colaboradores y colegas que llevan adelante una experiencia en común..."

El análisis anterior confirma que hay dos dimensiones de la Innovación, una de carácter industrial que implica una dimensión pública, y otra privada del conocimiento y la tecnología. Además, se puede hacer una distinción entre la tecnología y la información: mientras la información trasciende los límites de las empresas, la tecnología implica un conocimiento tácito y específico que no es ni puede ser registrado por escrito en proyectos y planos y que, por ende, no puede ser difundido exhaustivamente, ni como información pública ni como información patentada o de propiedad exclusiva. El hecho de que dicho conocimiento tácito resida en los individuos, las organizaciones y las redes es de particular importancia.

Donde las empresas que compiten entre sí están sólidamente integradas en una red formada por otras empresas, instituciones e infraestructuras, pueden capturar las externalidades y el conocimiento que se generan en la misma red. La apropiación de las externalidades y la difusión de conocimiento en un grupo restringido de empresas permitirán que las mismas muestren funciones de costo medio decreciente. Como consecuencia de lo anterior, las empresas compiten según su capacidad para capturar las interrelaciones tecnológicas, las economías de escala en la producción, y según su aptitud para incorporar el aprendizaje colectivo a través de la interacción y la distribución de conocimientos. Ese patrón de competencia define barreras de entrada a la red diferentes para empresas distintas, según la ventaja competitiva que cada una de las respectivas redes pueda desarrollar en términos de su función de costo decreciente y su escala de producción.

Todos los modelos contemporáneos de relación Universidad - Sociedad se basan en la necesidad de trabajar en Redes. Por tanto los Centros Universitarios Municipales (CUM), para cumplir su misión, necesitan realizar su trabajo en redes cuyos actores sean el Gobierno Local, las otras Sedes Universitarias del territorio, las Bibliotecas y Centros de Información, las empresas, los representantes locales de Ministerios como el Ministerio de Agricultura, las organizaciones políticas, sociales y profesionales y los movimientos sociales como el Forum de Ciencia y Técnica y las ONG. Cada uno de estos actores tiene diferente función dentro de la Red pero todos son importantes.

La Extensión Universitaria, como Gestión del Conocimiento en este contexto, consiste en gran medida en colaborar en la identificación de problemas locales que requieran del conocimiento para su solución y contribuir a identificar las organizaciones o personas que pueden aportarlo para luego construir los nexos, las redes y los flujos de conocimientos que permitan la asimilación, evaluación, procesamiento y uso de esos conocimientos.

La Extensión Universitaria del conocimiento a nivel local plantea retos de gran complejidad: Frecuentemente los problemas son complejos y necesitan un abordaje multidisciplinario, requieren integración de disciplinas en la búsqueda de respuestas cuya solución se logra muchas veces combinando inteligentemente los conocimientos existentes.

Generalmente el conocimiento requerido está integrado a la práctica, se necesita para resolver un problema y buena parte de él existe por lo que solo es necesario transferirlo con creatividad teniendo en cuenta la singularidad de las circunstancias locales. 
Existe un fuerte nexo entre innovación y aprendizaje por lo que para introducir lo nuevo antes hay que capacitar al personal que trabajará en el proceso.

De acuerdo con Lage (2005) el "Conocimiento Relevante" producido a nivel local se debe caracterizar por ser: Colectivo (incorporado a las organizaciones), Combinatorio (de fuentes y disciplinas diversas), Concreto (vinculado a la solución de problemas específicos), Tácito (frecuentemente no estructurado) y Local. Esa definición ilustra muy bien el tipo de conocimientos que deberán gestionar los Centros Universitarios Municipales para atender los problemas del territorio que las rodea. En la Gestión del Conocimiento a nivel local se deben eliminar las distancias que artificialmente se han creado entre las disciplinas, entre los actores y entre los procesos de aprendizaje, superación, capacitación, investigación e innovación. Esto es muy importante porque obliga a trabajar integradamente a todos los actores en función de la obtención de un conocimiento utilizable.

Lage (2005) ha resumido los pasos de la Gestión del Conocimiento como Extensión Universitaria en: a) Identificación de los actores, b) Construcción de redes, c) Construcción de la conectividad, d) Estimulación y organización de interacciones, e) Creación de capacidad de asimilar conocimientos y tecnologías, f) Armar "ciclos cerrados" a través de Dirección por Proyectos, g) Implementación de la "Capacitación para toda la vida", h) Seleccionar, capacitar y evaluar los cuadros; i) Construir infraestructura informática con conectividad y redes) y j) Construir y evaluar indicadores de desempeño.

En el contexto de una situación tan complicada y cambiante a todos los niveles tenemos que ponernos a tono con las complejidades de la vida económica, política y social del territorio para responder a sus crecientes exigencias.

Esta situación llevó a plantear la Hipótesis de que: El trabajo en Red puede realizar un aporte sustantivo al Desarrollo Agropecuario Local de un Municipio cubano.

Por eso el objetivo general de este trabajo es: Fundamentar el rol de la Red Local de Gestión Universitaria del Conocimiento y la Innovación (GUCID) como base del Desarrollo Local en un municipio cubano.

\section{METODOLOGÍA}

Se realizó un estudio sobre el trabajo de las formas productivas de la agricultura de Camajuaní, Villa Clara, Cuba. Seis equipos de trabajo se distribuyeron por el municipio para visitar fincas y aplicar instrumento de Categorización de Fincas. Compuestos por 2 miembros del Grupo Provincial de Apoyo al Movimiento Agroecológico, 2 miembros del Grupo Municipal de Apoyo al Movimiento Agroecológico, 1 representante del CUM, alumnos del CUM que viven en la zona, 1 representante de la Delegación Municipal de la Agricultura y 1 directivo local del Buró de la ANAP. Dirigieron el trabajo la Presidenta de la ANAP provincial, la Jefa del Grupo Provincial de Apoyo al Movimiento Agroecológico y el Presidente de la ANAP Municipal. Televisión Comunitaria documentó el proceso.

Se visitaron 36 fincas en 12 Cooperativas del municipio y 43 fincas de 14 CCS y CPA. Para la realización del diagnóstico se siguió la guía a la que se le da respuesta en relación con la finca objeto de diagnóstico. La misma mide 15 Indicadores Integrales que se detallan a 
continuación:

\section{4 indicadores sociales:}

-Participación en Movimiento Agroecológico Campesino a Campesino y la ANAP: 10 puntos -Calidad de vida: 10 puntos

-Equidad de género: 10 puntos

-Reafirmación de la identidad campesina: 10 puntos

\section{1 indicadores agroecológico - productivos:}

-Conocimiento y Compromiso Agroecológico: 10 puntos

-Calidad de insumos producidos en las fincas: 10 puntos

-Revalorización y Aprovechamiento de recursos: 10 puntos

-Agrobiodiversidad: 10 puntos

-Manejo del microclima: 10 puntos

-Producción de semilla: 10 puntos

-Manejo ecológico de la fertilidad del suelo: 10 puntos

-Manejo ecológico de la conservación del suelo: 10 puntos

-Manejo ecológico de plagas: 10 puntos

-Presencia de policultivos: 10 puntos

-Interrelación Producción - Sociedad - Alimentación: 10puntos

-Agroindustria Artesanal: 10 puntos

Terminada la evaluación de cada finca son asentados los resultados obtenidos en cada punto evaluado en el modelo. Se suma la cantidad de puntos obtenidos por la finca y se divide entre 15 para calcular el promedio de puntos obtenidos por indicador.

La clasificación de la finca se hace por la Escala: Categoría I: 4,0 a 6,9 puntos. Categoría II: 7,0 a 8,9 puntos. Categoría III: 9,0 a 10,0 puntos.

También se trabajó en 28 Unidades de Base de la Agricultura del municipio, entre septiembre y noviembre de 2009 con estudiantes de Ingeniería Agropecuaria del CUM para lograr una caracterización y diagnóstico de cada unidad mediante la evaluación de la evolución y composición de las diferentes producciones evaluando el incremento de los rendimientos por $\mathrm{m}^{2}$. Para la realización del diagnóstico se siguió la guía a la que se le da respuesta en relación con la unidad objeto de diagnóstico. Como resultado del análisis de la información obtenida se elaboró el banco de problemas de la Agricultura en el Municipio. El diagnóstico constituyó una herramienta para el análisis del proceso productivo por la Delegación del Ministerio de la Agricultura y el Gobierno.

\section{Se siguieron los pasos del Diagnóstico:}

- Clasificación de los suelos, sus características en función del desarrollo vegetal.

- Principales agentes de la erosión de los suelos.

- Medidas de conservación de los suelos en función de la erosión, salinidad y otros.

- Clasificación agro-productiva de suelos. Análisis de sus características agro-productivas.

- Costo unitario de los diferentes cultivos. Resultados económico - financieros de la unidad.

- Análisis de cómo se pone de manifiesto la integración agricultura- ganadería.

- Preparación de suelo. Equipos e implementos que se utilizan, tipos y calidad de las labores.

- Evaluación de la siembra y plantación. Labores fitotécnicas específicas paras cada cultivo.

- Cosecha, momento y método, manipulación, conservación y comercialización del pro- 
ducto

- Masa Porcina. Masa Ovina. Masa Bobina. Masa de Aves de Corral.

- Actividad mecanizada que efectúa la Unidad y consumo de combustible según la labor.

- Tipos de implementos. Máquinas Agrícolas. Guía del control de los mantenimientos.

- Problemas técnicos que presentan los tractores, implementos y máquinas agrícolas.

\section{Diagnóstico sobre Sanidad Vegetal.}

- Cultivos principales en la entidad, plagas y malezas por cultivo y cómo es su combate.

- Características de los principales plaguicidas químicos que se utilizan en la entidad.

- Manipulación de los plaguicidas químicos. Conservación de los plaguicidas químicos.

- Principales productos plaguicidas biológicos que se utilizan en la entidad.

- Análisis sobre protección del medio ambiente en el ecosistema donde se ubica la entidad.

A los datos obtenidos se les aplicaron instrumentos gerenciales para definir los diagnósticos. Se empleó la Matriz de Vester para mostrar la interacciones de dependencia y motricidad entre las variables identificadas y comprobar el grado en que cada variable afecta a las otras. Para ello se listaron los problemas relevantes detectados en el diagnostico en filas y columnas en un mismo orden y se les asignaron valores de categoría a través de una escala. Con los resultados de esta matriz se clasificaron las variables para el trabajo corto y a mediano plazo. Nivel de causalidad en base la escala. 0- No es causa. 1- Causa indirecta. 2- Causa medianamente directa. 3- Causa muy directa.

El Diagrama de causa y efecto permitió realizar un análisis de las causas que inciden en la situación productiva, identificando los problemas humanos, tecnológicos, agrobiológicos y materiales que deben solucionarse.

La Matriz DAFO: En ella se reflejan componentes internos que constituyen las fortalezas y debilidades de la unidad, que proceden de la dotación de recursos, estructura y comportamiento y los agentes que actúan. Entre los componentes externos analizamos las oportunidades y amenazas existentes en el entorno que pueden incidir sobre cada unidad.

\section{RESULTADOS}

\section{El diagnostico participativo en las unidades productivas arrojó que:}

- El $62 \%$ del área agrícola se cataloga de poco productivo y el $48 \%$ de muy poco productivo, motivado por mal drenaje, compactación de suelo y utilización incorrecta de la agrotecnia.

- $\quad$ El 30\% del área total estaba formada por terrenos arcillosos y pedregosos.

- En ciertas épocas del año existe superproducción de alimentos, escaseando en otra época.

- Solo $17 \%$ del área y $23.4 \%$ en faja reguladora en ríos, arroyos y embalses está reforestada.

- Afectadas especies de fauna endémica como zunzún, pájaro carpintero y paloma rabiche.

- Falta de conocimiento por campesinos, familiares y comunidad sobre medidas y técnicas para protección y conservación del medio ambiente de amplio empleo. 


\section{Entre los problemas fundamentales encontrados en algunos predios están:}

- No sistematicidad en aplicación de Materia Orgánica y poca área de lombricultura y compost.

- Deficiente intercalamiento de cultivos e Insuficiente empleo de injertos.

- Poco uso de medios biológicos para el control de plagas y enfermedades.

- Afectación con pastos naturales en la sequía crea situación desfavorable para los animales.

- Aún emplean productos químicos contra plagas y enfermedades con afectación al medio.

- Baja calidad genética y fitosanitaria de algunas semillas.

- Poca apicultura.

\section{Al consolidar el Municipio se pudo ver un grupo de Problemas Generalizados:}

1. Ausencia de personal calificado y Mala Vinculación del hombre al área, (Subjetivo)

2. No aplicación de Pago por Resultados y Falta de Estimulación moral y material. (Subjetivo)

3. Problemas de Capacitación a todos los niveles. (Subjetivo)

4. Mala calidad y eficiencia de los Convenios con Acopio. (Subjetivo)

5. Problemas de riego y drenaje e Indisciplina tecnológica generalizada. (Objetivo)

6. Falta de un plan de acciones que trace las políticas a seguir en cada unidad. (Subjetivo)

7. Insuficiente uso de materia orgánica y producción de humus y compost para elevar los rendimientos como alternativa de fertilización. (Subjetivo)

8. Insuficiente uso de la producción de medios biológicos del CREE. (Subjetivo)

9. Baja rentabilidad de las unidades. (Consecuencias)

Como se puede observar la mayoría tienen un carácter subjetivo y dejan al descubierto incumplimientos de los Lineamientos del VI Congreso del Partido que deben ser prioritarias en la dirección de la economía cubana.

\section{Resultados de diagnósticos}

- No se exige nivel cultural y científico a directivos que administran grandes recursos y sobre los que recaen un grupo de responsabilidades y decisiones que toman sin conocimiento.

- Pobre funcionamiento de los Consejos Técnicos Asesores y las Comisiones de Cuadros.

- $\quad$ No está garantizado el relevo y preparación del personal que se jubila.

- Los puestos directivos no se cubren con profesionales afines con el requisito del cargo.

- No correspondencia del perfil de graduado del técnico medio y las funciones que realiza.

- No existe actualización de conocimientos y manejo de las nuevas tecnologías para el personal que ha laborado por más de 2012 años en la actividad.

- Las plantillas de cargos no se basan en las necesidades de las empresas, en general se adecuan los requisitos al hombre que está ocupando el cargo.

- El personal no está preparado para cumplir con estudios la organización del trabajo y Recursos Humanos, carga y capacidad y productividad.

- Desmotivación salarial. Muchos reciben salarios de un profesional graduado sin siquiera estar estudiando y sin cumplir los requisitos de las plazas.

\section{Teniendo en cuenta este diagnóstico se elaboraron los siguientes programas:}

a) Programa para la conservación y rehabilitación de los suelos: Debido al uso irracional y a la explotación indiscriminada que se le ha dado a nuestros suelos los mismos han reducido sus reservas de nutrientes. Se ha capacitado en la existencia de plantas beneficiosas para los suelos por los aportes de nutrientes que hacen. Entre ellas se encuentran las leguminosas que garantizan fijación biológica de Nitrógeno en suelo. Se usan plantas con gran 
producción de follaje en barbecho para su incorporación en la preparación del suelo y para la conservación de estos suelos se establecieron un grupo de medidas:

- No roturar tierra a favor de la pendiente sino por el trazado de las curvas de nivel para evitar el arrastre erosivo.

- No quemar residuos de cosecha. Destruye capa vegetal y provoca calentamiento global.

- Crear barreras para contener el agua y favorecer la infiltración, minimizando la erosión.

- La rotación de los suelos para diversificar los cultivos en una misma área (desfasados en época de siembra, cosecha y de tiempo requerido para la preparación del suelo). Ej.: Planta que aporte Nitrógeno al suelo genera fertilidad para cultivo extractivo posterior (yuca, maíz).

- La asociación de cultivos comprende la combinación de al menos dos cultivos. Ej.: fríjolboniato, maíz-boniato-fríjol, fríjol-girasol, maíz-plátano, maíz-maní, cítricos-boniato, cítrico-calabaza. Esas combinaciones permiten regulación de malezas y hacen uso racional de suelo y agua, disminuyen incidencia de plagas y enfermedades y permiten mayor producción.

- Empleo de tracción animal en las labores. Bueyes, yugos e implementos son elementos fundamentales en la preparación del suelo. Actualmente se utilizan arados, gradas y cosechadoras. La utilización de maquinarias contribuye a la compactación del suelo.

- Usar excretas animales como abono orgánico baja carga contaminante y favorece fertilidad.

b) Programa para la protección de la biodiversidad: Ha logrado concientización de la necesidad de implementación de una agricultura agroecológica y sostenible.

- Cada forma productiva creó un vivero de árboles frutales y maderables para llevar a cabo la reforestación. Entre las plantas más propagadas están los cítricos, árboles maderables y frutales que son usados como postes vivos, e intercalamiento de árboles con otros cultivos.

- Se tuvo como resultado que toda la faja hidroreguladora en ríos, arroyos y embalses están reforestadas y el área boscosa ascendió de un $17 \%$ a un $27 \%$, previéndose llegar al $30 \%$.

- Al aumentar la zona boscosa aumenta también el anidamiento de las aves, observándose recuperación de las poblaciones de especies de aves en peligro de extinción como: el zunzún, pájaro carpintero y paloma rabiche.

c) Programa de conservación de alimentos: Al detectar superproducción de frutas en ciertas estaciones del año, fue necesario crear un grupo llamado Consejo de Mayores con el objetivo de capacitar mediante talleres para elaborar conservas de alimentos.

Ejemplo: En frutas como mango, tomate y guayaba se hacen pulpas para todo el año. Para esto contamos con el centro de elaboración ubicado en el Consejo Popular Taguayabón que se construyó por la cooperación de todos los campesinos y equipado con financiamiento del Proyecto HIVOS.

\section{d) Programa de Educación Ambiental para la Comunidad}

- Se organizó el I Taller Nacional de Educación para la comunidad. Este incluyó muestras del trabajo de todos los factores y actores de la sociedad. Los campesinos y sus familiares ayudan a rehabilitación de suelos, a la reforestación y al cuidado y aumento de las especies.

- Están inmersas las organizaciones primarias de salud, responsables de la potabilidad del agua para el consumo, luchar contra la propagación del SIDA y ayudar al mejoramiento humano.

- La Comunidad tienen como consigna Mi Patio Productivo y Mi Jardín Florido incentivando 
a tener en los patios más de seis especies de árboles frutales, maderables y hortalizas.

- El Promotor Cultural ayuda a la conservación y promoción de las tradiciones.

- El Consejo de Mayores: grupo que se encarga de promover la utilización de la cocina vegetariana, alcanzar una alimentación y vida sana. Para eso educa a la comunidad y con su apoyo se puede alcanzar una agricultura y medio ambiente sano y limpio.

- La Asociación de Combatientes ayuda a la divulgación de los hechos históricos más importantes relacionados con la naturaleza.

- Los niños de las Escuelas de las Cooperativas se preparan para cuidar el medio ambiente. Realizan visitas al Bosque Martiano donde se les enseña a apreciar y cuidar la flora y la fauna, haciéndoles llegar el amor y el pensamiento ecologista de José Martí.

- El programa de promoción "Campesino a Campesino" que surgió en la provincia de Villa Clara en 1997, auspiciado por la ONG Pan para el Mundo, comienza a promoverse en la CCS Benito Ramírez en el año 2000 con cinco promotores y un facilitador. El objetivo de este movimiento agroecológico es alcanzar la rehabilitación de más del $50 \%$ de las tierras cultivadas y que más del $50 \%$ de los campesinos de la CCS se conviertan en promotores para poder satisfacer las necesidades de esta generación sin afectar a las venideras.

Los estudios socioculturales concluyeron que la lentitud con que en el territorio se han introducido las tecnologías agroecológicas obedece a cuatro factores: Culturales: no hay tradición y el campesino es aferrado a lo tradicional, Educativos: Debemos estar concientes y preparados para implementar estas tecnologías, Sociales: El trabajo agrícola es duro y los jóvenes no se sienten atraídos por él al disponer de otras opciones como el estudio. Por otro lado la dedicación y la entrega total que requiere y Económicos: La introducción de estas tecnologías requiere de recursos que el país no siempre tiene disponibles o no están al alcance de los productores.

- El Know how generado en el trabajo con los Proyectos de Agricultura Sostenible es:

- Mamey que fructifica en 3 años mediante tipos de injertos y ajustes a las fases de la luna.

- Variedad de fríjol de 9 granos por vaina obtenidas por selección de los campesinos.

- Asociación con Empresa Mecánica para diseño de prototipos de implementos agrícolas.

- Conservación de semillas en frascos sumergidos en agua del arroyo. Emerio Reyes.

- Extracción del Guano de murciélago y uso como fertilizante orgánico. Isidro Oliva Pérez.

- Uso del látex del Cardón como pesticida biológico. Cooperativa Abel Santa María

- Uso de restos del despalillo (Palo de tabaco) como cobertura. CCS Abel Santa María

- Sistemas de venta al estado de todos los productos. Ajo. BANDEC.

- Consejo de Ancianos gestiona conocimiento ancestral campesino. CCS Benito Ramírez.

- Preparar Conservas en períodos pico de cosecha para evitar pérdidas. CPA 13 de marzo.

- Ferias de generalización de resultados. Proyecto PIAL y CIAP.

- Extractos de plantas repelentes en Manejo Integrado de Plagas de Cultivos. Alexey Cruz.

\section{CONCLUSIONES}

El modelo de trabajo en red es un elemento importante para explicar cómo se configuran los Sistemas de Innovación Local en la praxis. 
en redes cuyos actores sean el Gobierno Local, Centros de Información, empresas, representantes locales de Ministerios como la Agricultura, las organizaciones políticas, sociales y profesionales y los movimientos sociales.

La Extensión Universitaria desde el CUM para introducir las tecnologías agroecológicas en la Agricultura inició su desarrollo económico y ecológico sustentable, por lo que las unidades productivas muestran un uso más intensivo del recurso tierra, expresado en el número de especies manejadas así como el contenido de materia orgánica.

Esta investigación demostró que el rol de la Red de Gestión Universitaria del Conocimiento y la Innovación en el municipio es interactuar con el sector productivo y de servicios para diagnosticar los problemas que necesitan de ciencia para su solución y hacerlos saber a los decisores para que tomen sus decisiones en función del desarrollo local.

\section{AGRADECIMIENTOS}

Este trabajo fue dirigido y financiado como resultado de los Proyectos 3.040. "Centro de capacitación y concientización sobre desarrollo sostenible de los actores del proyecto Agenda 21 en el entorno de la CUM Camajuaní" y 3.043. "Servicios de extensión de la SUM Camajuaní a la localidad", del Programa Ramal MES - GUCID y del Proyecto 7.06. Sistema de acciones para caracterizar desde el punto de vista social al municipio de Camajuaní y para establecer propuestas que conduzcan a su desarrollo, del Programa Ramal MES.

\section{REFERENCIAS}

ALBURQUERQUE, Francisco. Cambio Tecnológico, Globalización y Desarrollo Económico Local. Madrid: Instituto de Economía y Geografía, Consejo Superior de Investigaciones Científicas. 1998. 183 p.

CIMOLI, Mario. Redes, estructuras de mercado y shocks económicos. CEPAL- GTZ. En: Cimoli, M. Cambios estructurales de los sistemas de innovación en América Latina. Redes Jerarquías y Dinámicas Productivas. Santiago de Chile: Naciones Unidas. 2005. pp. 123-139.

CIMOLI Mario and DE LA MOTHE John. The Governance of Technology and Development. En: Science, Technology and Governance. London: Pinter Publisher. 2001. pp. 2-15.

DE BRESSON, C. and AMESSE F. Networks of Innovation: a Review and Introduction to the Issues. RESEARCH POLICY, 20(5): 363-381. 1991.

DOSI, G., TYSON, L. and ZYSMAN, J. Trade, Technologies and Development: framework for discussing Japan. En: Politics and Productivity. New York: Ballinger Publishing. 1990. pp. 45-63. 
DOSI, G. Some Notes on National Systems of Innovation and Production, and their Implication for Economic Analysis. En: ARCHIBUGI D, et al. (Eds.) Innovation Policies in a Global Economy. London: Cambridge University Press. 1999. pp. 67-85.

FREEMAN, Charles. Technological Revolution and Catching up: ITC and the NICs. En: FAGERBERG, J., VON TUNZELMAN, N., VERSPAGEN, B. The Dynamics of Technology, Trade and Growth. London: Edgar Elgar. 1994. pp. 32-54.

HIRSCHMAN, A. Generalized linkage approach to development, with special reference to staples. En NASH, M. Essays on Economic Development and Cultural Change in Honor of B. F. Hoselitz. Chicago: University of Chicago Press. 1977. pp. 85-97.

MISA, T. Constructive Technology Assessment: Cases. Concepts, Conceptualization. Twente, The Netherlands: Conference on Constructive Technology Assessment. 1991. pp. 8-19.

NELSON, R. The Agenda for Growth Theory: a Different Point of View, CAMBRIDGE J. ECON., (22): 497-520. 1998.

POLANY, M. The Tacit Dimension. New York: Doubleday Anchor. 1967. pp. 85-98.

SOETE, L. The Challenges of Innovation. Seville: IPTS Report. 1996. pp. 16-23. 